\title{
FINITE FRATTINI FACTORS IN FINITELY GENERATED SOLUBLE GROUPS ${ }^{1}$
}

\author{
JOHN C. LENNOX
}

ABstract. Results of Philip Hall are used to prove that subgroups of finitely generated Abelian-by-nilpotent groups are finite if their Frattini factor groups are finite.

1. Introduction. In this paper we consider finitely generated soluble groups and their subgroups in relation to the question whether they are finite if their Frattini factor groups are finite. By the Frattini factor group of a group $G$ we mean $G / \Phi(G)$ where $\Phi(G)$ is the Frattini subgroup of $G$.

First of all we have the following result which gives a positive answer in the case of finitely generated soluble groups.

THEOREM A. Finitely generated soluble groups with finite Frattini factor groups are finite.

It would be of independent interest to know whether there exist finitely generated groups of infinite order with finite Frattini factors. We note that this problem is easily seen to be related (see Theorem $\mathrm{A}^{*}$ below) to the question of the existence of groups with finitely generated yet nonnilpotent Frattini subgroups.

Turning now to subgroups of finitely generated soluble groups it is clear that a different situation arises here since such subgroups need not be finitely generated. In fact Neumann and Neumann in [7] showed that any countable soluble group of derived length $d$ can be embedded in a 2-generator soluble group of derived length less than or equal to $d+2$. Furthermore Hall showed in [4] that any given countable Abelian group $C$ can be embedded as the centre of a finitely generated centre-bymetabelian group. Hence, taking $C$ for example to be a quasi-cyclic $p$-group ( $p$ a prime), we see that it is possible for an infinite Abelian subgroup of a finitely generated centre-by-metabelian group to have trivial Frattini factor group. Thus subgroups of finitely generated soluble groups are not in general finite if their Frattini factors are finite.

Received by the editors September 25, 1972 and, in revised form, November 20, 1972. AMS (MOS) subject classifications (1970). Primary 20E15; Secondary $20 \mathrm{E} 20$.

Key words and phrases. Frattini subgroup, finitely generated soluble group.

1 Thanks are due to the referee for several helpful suggestions.

(c) American Mathematical Society 1973 
In view of Hall's methods and results in the paper mentioned above it is natural to consider next the class of all finitely generated Abelian-bynilpotent groups. We shall prove

THEOREM B. Subgroups of finitely generated Abelian-by-nilpotent groups are finite if their Frattini factor groups are finite.

It follows from Theorem $B$ and the preceding remarks that the property of subgroups being finite whenever their Frattini factors are finite is an $\mathfrak{U} \mathfrak{N}$-property in the sense of Hall [5, p. 602].

\section{Proofs.}

Proof of Theorem A. Theorem A is an immediate consequence of the somewhat stronger

THEOREM A*. Suppose $G$ is a finitely generated group with an ascending series with finite or locally soluble factors. Then $G$ is finite if $G / \Phi(G)$ is finite.

Suppose then that $G$ satisfies the hypothesis of Theorem $A^{*}$ and $G / \Phi(G)$ is finite. We assume $G$ is infinite and produce a contradiction. We first of all reduce the problem by means of an argument similar to that of Baer [2, §2, Lemma 4]. Let $N_{\lambda}, \lambda \in \Lambda$, be a chain of normal subgroups of $G$ with $G / N_{\lambda}$ infinite for all $\lambda \in \Lambda$. Let $N$ be the union of the $N_{\lambda}, \lambda \in \Lambda$. If $G / N$ is finite then, since $G$ is finitely generated, $N$ is finitely generated. Hence $N=N_{\lambda}$ for some $\lambda \in \Lambda$, which contradicts the fact that $G / N_{\lambda}$ is infinite. Hence $G / N$ is infinite and we may deduce from Zorn's Lemma that there exists a normal subgroup $M$ of $G$ maximal with respect to $G / M$ infinite. We may assume $M=1$ and therefore that every proper homomorphic image of $G$ is finite.

Now let $\left\{G_{\alpha}\right\}$ be the given ascending series for $G$. Then there is a first ordinal $\beta$ for which $\left|G: G_{\beta}\right|$ is finite. Since $G_{\beta}$ is finitely generated, $\beta$ cannot be a limit ordinal. So $\beta-1$ exists and $G_{\beta} / G_{\beta-1}$ is infinite by definition of $\beta$. It follows that $G_{\beta} / G_{\beta-1}$ must be locally soluble and hence soluble since $G_{\beta}$ is finitely generated. If $C$ is the core of $G_{\beta-1}$ in $G$ then, clearly, $G / C$ is soluble-by-finite. If $C \neq 1$ then $G / C$ is finite and so $\left|G: G_{\beta-1}\right|$ is finite, a contradiction. Therefore $C=1$ and $G$ is soluble-by-finite. Hence $G$ has a nontrivial Abelian normal subgroup $A$ and $G / A$ is finite. Hence $G$ is Abelian-by-finite. But this means that $G$ has a finitely generated Abelian subgroup of finite index and it follows from a result of Hall [5, Lemma 10] that $\Phi(G)$ is finite. However $G / \Phi(G)$ is finite and hence $G$ is finite, a contradiction.

Proof OF TheORem B. The proof of this result depends on properties of the countable Abelian groups which Hall in [5, p. 605] calls $\mathfrak{B}$-groups. 
An Abelian group $B$ belongs to the class $\mathfrak{B}$ if and only if it can be extended to a finitely generated group $G$ such that $G / B$ is polycyclic. The properties of $\mathfrak{B}$-groups which we need are

Lemma 1 [5, Lemma 8]. Every $\mathfrak{B}$-group B satisfies the maximal condition on characteristic subgroups, the torsion subgroup $T$ of $B$ is of finite exponent and is a direct product of cyclic groups and $B=T \times N$ where $N$ is a torsion-free $\mathfrak{B}$-group

and

Lemma 2 [5, Lemma 12]. Let $B$ be a torsion-free $\mathfrak{B}$-group and let $\pi$ be any infinite set of primes. Then $\bigcap_{p \in \pi} B^{p}=1$.

Suppose now that $G$ is a finitely generated Abelian-by-nilpotent group and that $H$ is a subgroup of $G$ with $H / \Phi(H)$ finite but $H$ is not finite. If $N \triangleleft G$ and if bars denote factors mod $N$ then clearly $\bar{H} / \Phi(\bar{H})$ is also finite. Therefore since $G$ satisfies the maximal condition on normal subgroups [4, Theorem 5], we may assume that $H N / N$ is finite for all $1 \neq N \triangleleft G$. (We remark that this last condition on its own is trivially insufficient to ensure the finiteness of a subgroup $H$, e.g. take $H=G$ to be an infinite cyclic group.)

By definition there exists a normal Abelian subgroup $A$ of $G$ such that $G / A$ is finitely generated nilpotent. If $A=1$ then $G$, and therefore $H$, is finitely generated nilpotent and hence $H$ is finite by Theorem $\mathrm{A}$, a contradiction. Therefore $A \neq 1$ and $H A \mid A \cong H / H \cap A$ is finite. Thus $H$ is Abelian-by-finite. Furthermore since $H / H \cap N$ is finite for all $1 \neq N \triangleleft G$ and $H$ is infinite it follows that $G$ can contain no pair of normal subgroups distinct from 1 and with intersection 1 . Since $A$ is a $\mathfrak{B}$-group it follows easily from Lemma 1 that either

(i) $A$ is periodic of exponent $p^{m}$ for some prime $p$ and positive integer $m$, or

(ii) $A$ is torsion-free.

We treat these two possibilities separately.

Case (i). A is of exponent $p^{m}$ for some prime $p$.

In this case $A$, and therefore $H \cap A$, is a direct product of cyclic groups of $p$-power order. The required contradiction in this case follows at once from

LEMma 3. Let $K \triangleleft H$ where $K$ and $H / K$ are nilpotent. Then $H$ is finite if and only if $K$ is periodic, $H / \Phi(H) \cap K$ is finite and the centre of $K$ is reduced.

As stated Lemma 3 is more general than we really need, but in this generality it may be of some independent interest. 
Proof of Lemma 3. Clearly only the sufficiency is in question. To establish it we first reduce to the case $K$ Abelian. Accordingly let $c$ be the nilpotency class of $K$ and suppose that we have dealt with the case $c=1$. Write $Z$ for the centre of $K$. Since $Z$ is reduced, so is every upper central factor of $K$ (see [8, Lemma 6.16(i)]). Also $\Phi(H Z / Z) \geqq \Phi(H) Z / Z$ so that $H / Z$ inherits the properties of $H$ and therefore is finite by induction on $c$. Thus $K / Z$ is finite which, by a well-known theorem of Schur, implies that $K^{\prime}$, the derived subgroup of $K$, is finite. It is easy to see that $K / K^{\prime}$ is reduced and so $H / K^{\prime}$ is finite by the case $c=1$. Hence $H$ is finite and our reduction is complete.

Suppose, therefore, that $K$ is Abelian and that the case $K$ an elementary Abelian $p$-group ( $p$ a prime) has been dealt with. If $\pi$ is a set of primes write $K_{\pi}$ for the $\pi$-component of $K$. For any prime $p$ let $D=$ $K_{p}^{p} \times K_{p^{\prime}}$. Then $K / D$ is an elementary Abelian $p$-group and by hypothesis it is finite. Therefore $K_{p} / K_{p}^{p}$ is finite, say of order $p^{r}$. Suppose $K_{p}$ is infinite. Since $K_{p}$ is reduced it has a nontrivial cyclic direct factor (see [6, Theorem 9]). Successive applications of this fact yield

$$
K_{p}=\left\langle k_{1}\right\rangle \times\left\langle k_{2}\right\rangle \times \cdots \times\left\langle k_{r+1}\right\rangle \times L
$$

for certain $1 \neq k_{i}$ and $L \leqq K_{p}$. But this implies that $\left|K_{p}: K_{p}^{p}\right| \geqq p^{r+1}$, a contradiction. Therefore $K_{p}$ is finite for every prime $p$.

Let $\pi$ be the set of prime divisors of $|H: K| \cdot|H: \Phi(H)|$ and let $N=K_{\pi^{\prime}}$. Then $N \triangleleft H$ and $H / N$ is a finite $\pi$-group. Hence by Schur's theorem $H$ splits over $N$ so that $H=N X$ for some $X \leqq H$ with $N \cap X=1$. If $p \in \pi^{\prime}$ and $K_{p} \neq 1$ we may choose, since $K_{p}$ is finite, a maximal (proper) $H$-admissible subgroup $M(p)$, say, of $K_{p}$. It is then clear that $M(p) N_{p^{\prime}} X$ is a maximal subgroup of $H$ with index a power of $p$, so that $p$ divides $|H: \Phi(H)|$ and $p \in \pi$. Hence $K_{p}=1$, a contradiction. Therefore $N=1$ and $H$ is finite.

We are now left with the case $K$ an elementary Abelian $p$-group for some prime $p$. Let $L / K$ be the Sylow $p$-subgroup of the finite nilpotent group $H / K$. Then $L$ is an extension of an Abelian $p$-group of finite exponent by a finite $p$-group and therefore $L$ is nilpotent by a result of Baumslag [3, Lemma 3.8]. The hypotheses persist in $H / L^{\prime} L^{p}$. If $H / L^{\prime} L^{p}$ is finite then so is $L / L^{\prime} L^{p}$. Moreover $L / L^{\prime}$ is a direct product of cyclic $p$-groups and therefore we must also have $L / L^{\prime}$ finite. But $L$ is nilpotent and so $L$ is finite by a result of Baer [1]. Therefore $H$ is finite, as required. Hence we may assume that $L^{\prime} L^{p}=1$ so that $L$ is an elementary Abelian p-group. Moreover $H / L$ is a finite $p^{\prime}$-group. The fact that $H$ is finite now follows from the finiteness of $H / \Phi(H)$ together with

LemMa 4. Suppose $V$ is a normal elementary p-subgroup ( $p$ a prime) of a group $U$ and $U / V$ is a finite $p^{\prime}$-group. Then $\Phi(U) \cap V=1$. 
Proof. By Schur's theorem $V$ is complemented in $U$ by a $p^{\prime}$-group $T$, say, and by Maschke's theorem, $V$ is completely reducible as a $T$-module. Let $V=V_{1} \times \cdots \times V_{n}$ be a decomposition of $V$ into irreducible $T$-submodules and set

$$
W_{j}=\prod_{i \neq j} V_{i}
$$

Then $M_{j}=W_{j} T$ is a maximal subgroup of $U$ whence

$$
\Phi(U) \leqq \bigcap_{j=1}^{n} M_{j} .
$$

It now follows at once that $\Phi(U) \cap V=1$, as required. The proof of Case (i) is now complete.

Case (ii). A is torsion-free.

Here we need the following immediate corollary of Lemma 4.

Lemma 5. Suppose $V$ is a normal elementary Abelian subgroup of the finite group $U$ and $U / V$ is a $p^{\prime}$-group, p a prime. Then $|U| \leqq|U: \Phi(U)| \cdot|U: V|$.

Suppose, then, that $A$ is torsion-free and that $H / H \cap A$ and $H / \Phi(H)$ have the finite orders $l$ and $m$, respectively. Let $p$ be any prime not dividing $l$. Since $A$ is torsion-free $A^{p} \neq 1$ and therefore $\bar{H}=H / H \cap A^{p}$ is finite. Moreover $|\bar{H}: \Phi(\bar{H})| \leqq|H: \Phi(H)|$ and it follows from Lemma 5 that $|\bar{H}| \leqq l m$. We deduce that for infinitely many primes $p, H \cap A=H \cap A^{p}$ and so, by Lemma $2, H \cap A=1$. Hence $H$ is finite, a contradiction. The proof of Theorem $B$ is complete.

\section{REFERENCES}

1. R. Baer, Representations of groups as quotient groups. II. Minimal central chains of a group, Trans. Amer. Math. Soc. 58 (1945), 348-389. MR 7, 372.

2. ——, Nil-Gruppen, Math. Z. 62 (1955), 402-437. MR 17, 124.

3. G. Baumslag, Wreath products and p-groups, Proc. Cambridge Philos. Soc. 55 (1959), 224-231. MR 21 \#4179.

4. P. Hall, Finiteness conditions for soluble groups, Proc. London Math. Soc. (3) 4 (1954), 419-436. MR 17, 344.

5. - On the finiteness of certain soluble groups, Proc. London Math. Soc. (3) 9 (1959), 595-622. MR 22 \#1618.

6. I. Kaplansky, Infinite Abelian groups, Univ. of Michigan Press, Ann Arbor, Mich., 1954. MR 16, 444.

7. B. H. Neumann and H. Neumann, Embedding theorems for groups, J. London Math. Soc. 34 (1959), 465-479.

8. D. J. S. Robinson, Infinite soluble and nilpotent groups, Queen Mary College Math. Notes, London, 1967. MR 42 \#4635.

Department of Pure Mathematics, University College, Cardiff, Great BrITAIN 